\title{
Extramedullary hematopoiesis presenting as a compressive cord and cerebral lesion in a patient without a significant hematologic disorder: a case report
}

Amir Saied Seddighi ${ }^{1,3^{*}}$, Afsoun Seddighi ${ }^{2,3}$

\begin{abstract}
Introduction: Intracranial or spinal compressive lesions due to extramedullary hematopoiesis have been reported in the medical literature. Most of the reported cases are extradural lesions or, on rare occasions, foci within another neoplasm such as hemangioblastoma, meningioma or pilocytic astrocytoma. Often these cases occur in patients with an underlying hematological disorder such as acute myelogenic leukemia, myelofibrosis, or other myelodysplastic syndromes. Such lesions have also been reported in thalassemia major.
\end{abstract}

Case presentation: We report the case of a 43-year-old Iranian woman in whom extramedullary hematopoiesis presented as a compressive cord lesion and then later as an intracranial lesion.

Conclusions: To the best of our knowledge, we document the first reported case of sacral, lumbar, thoracic and cranial involvement in the same patient with extramedullary hematopoiesis, which seems both rare and remarkable.

\section{Introduction}

Intracranial or spinal involvement, manifesting as epidural lesions, due to extramedullary hematopoiesis $(\mathrm{EMH})$ is rare. The intracranial lesions are also reported as foci within another intracranial neoplasm such as hemangioblastoma, meningioma or pilocytic astrocytoma [1,2]. Extramedullary hematoopoiesis usually occurs in patients with a significant hematologic disorder like acute myelogenic leukemia (AML), myelofibrosis (MF), myelodysplastic syndromes or thalassemia major [1-3]. Prior to our case report and to the best of our knowledge, there has been no report of such a problem presenting as compressive spinal and cranial lesions in the same patient without a significant hematologic problem. The low incidence of EMH in central nervous system indicates that cells with hematopoietic potential find little supporting environment in CNS [3].

\footnotetext{
* Correspondence: a_sedighi@sbmu.ac.ir

'Shohada Tajrish Hospital, Beheshti University of Medical Sciences, Tehran, Iran
}

Full list of author information is available at the end of the article

\section{Case presentation}

Our case report, a 43-year-old Iranian woman, came to us complaining of back pain radiating to both of her lower extremities which had started two months previously. The pain was non-responsive to conventional medical treatments. In her general physical exam, mild splenomegaly was noted. A neuroexam showed decreased strength in both of her distal lower extremities (motor strength $=4 / 5$ ) and absent deep tendon reflexes in her lower extremities. She had a limping gait due to pain. A sensory exam indicated problems in the L4 and L5 dermatomes. Her sphincter function was normal. A lumbosacral magnetic resonance imaging (MRI) scan showed an abnormal para-vertebral mass extending from L3 to S3 which became enhanced after a contrast injection (Figure 1). In a dorsal spine MRI, another epidural lesion, extending from T3 to T11 with mild compression over the thoracic cord, was identified which became enhanced after a contrast injection (Figure 2). An MRI of both her cervical spine and brain were normal. An abdominal and pelvic computed tomography (CT) scan with and without intravenous and oral 


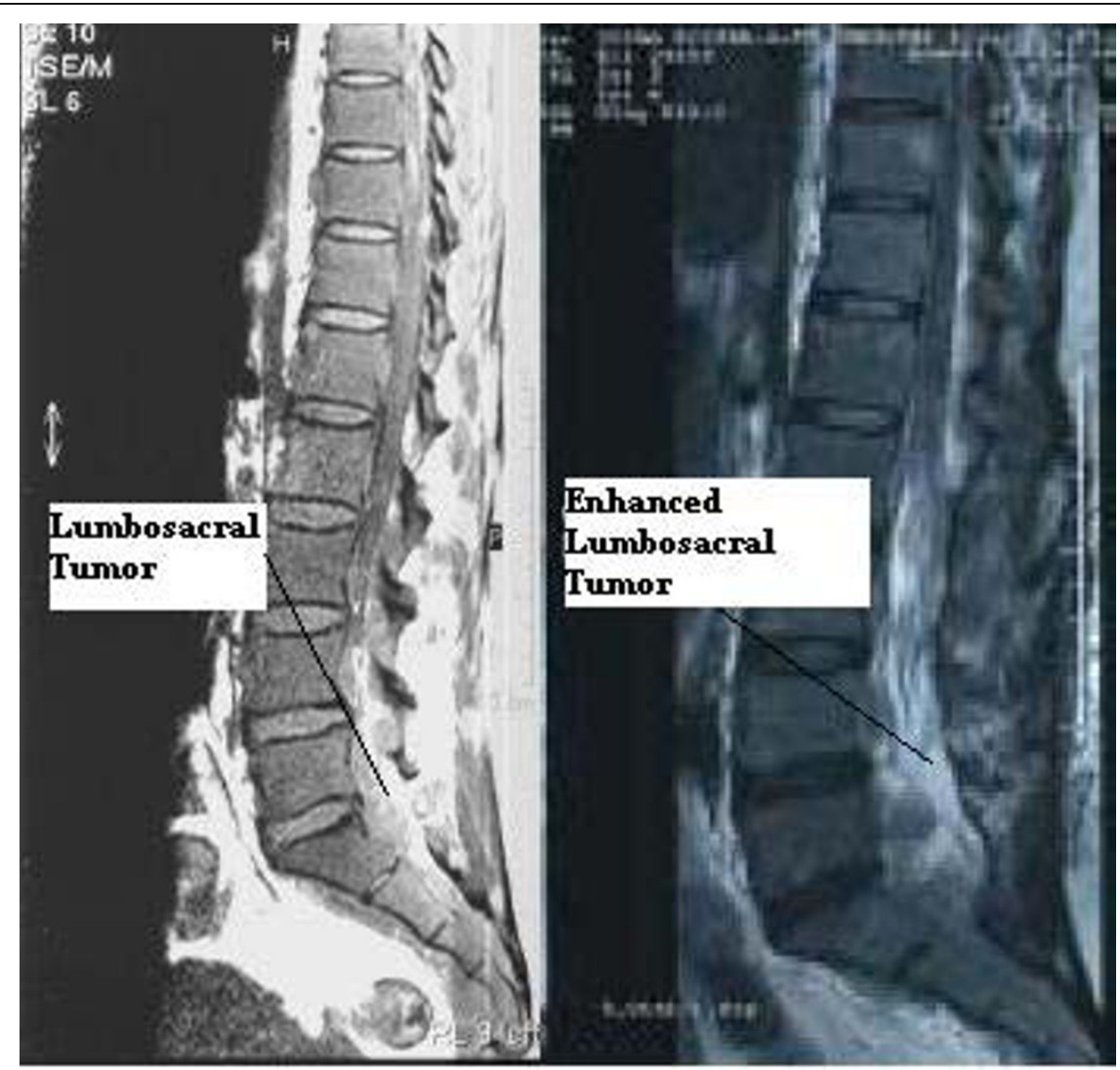

Figure 1 An MRI of the lumbosacral spine (left) showed an extradural lesion extending from L3 to S3 (left), which became enhanced after a contrast injection (right).

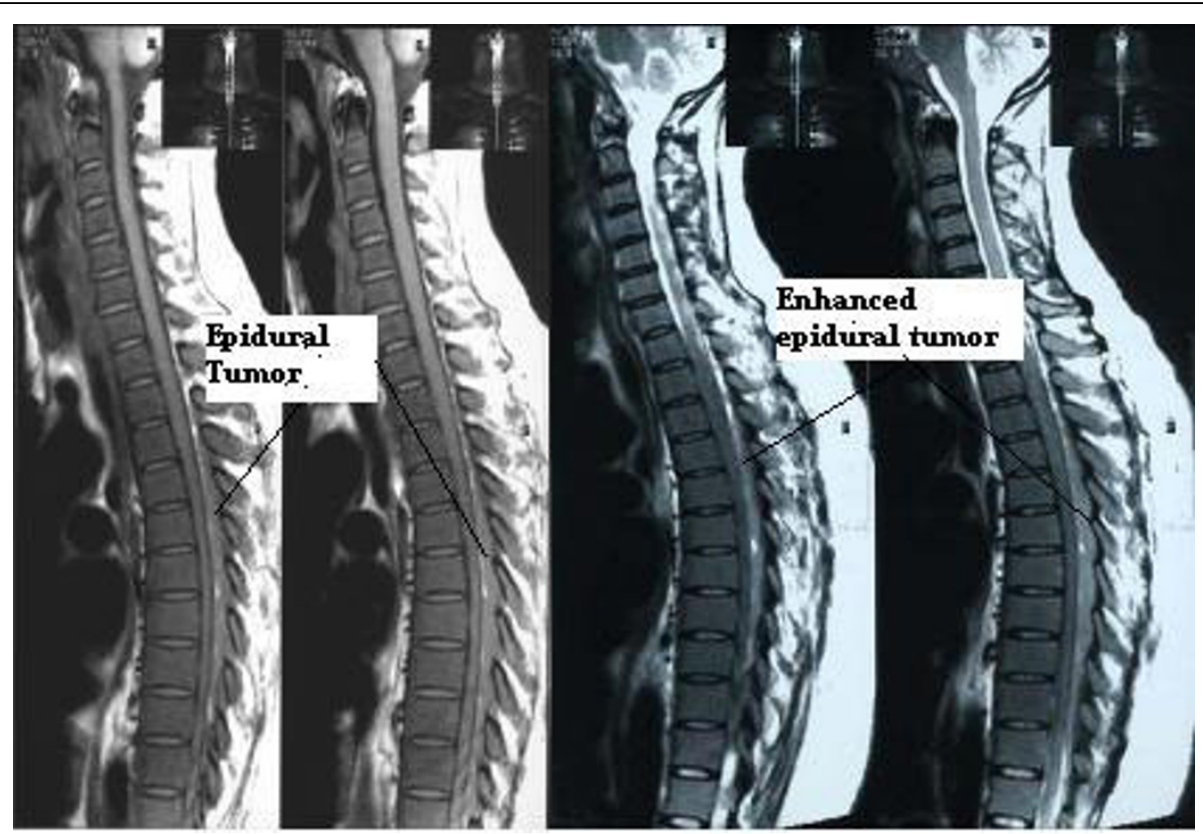

Figure 2 An MRI of the thoracic spine showed an extradural lesion extending from T3 to T11 (left) which became enhanced after a contrast injection (right). 
contrast media showed no abnormality. Para-clinical data showed an elevated erythrocyte count, decreased mean corpuscular volume (MCV), mean corpuscular hemoglobin $(\mathrm{MCH})$ and mean corpuscular hemoglobin concentration (MCHC). Her retic count was also elevated. Her white blood cell count showed mild leukocytosis and a few myelocytes and band cells.

Her red blood cell count was $7.66 \times 10^{6} / \mathrm{mm}^{3}$; her haemoglobin level was $20.5^{\mathrm{g}} / \mathrm{dl}$; her hematocrit level was 67.3\%; her mean corpuscular volume was $67 \mathrm{fl}$; her mean corpuscular hemoglobin level was $17.6 \mathrm{pg}$; her mean corpuscular hemoglobin concentration was $26.3^{\mathrm{g}}$ / $\mathrm{dl}$; her reticulocyte level was $3.5 \%$; her platelet count was $24,6000 / \mathrm{mm}^{3}$; her white blood cell count was 14.6 $\times 10^{3} / \mathrm{mm}^{3}$; the percentage of polmorphonuclear cells in her blood was $46 \%$; the percentage of monocytes was $2 \%$; the percentage of band cells was $1 \%$; the percentage of lymphocytes was $48 \%$; and the percentage of myelocytes was $3 \%$.

A consultation with the hematologist and a review of the peripheral blood smear yielded nucleated-RBC, anisocytosis $(1+)$, microcytosis $(2+)$, hypochromia $(2+)$, poikilocytosis $(2+)$, tear drop spherocytes and target cells (Figure 3). Her $\mathrm{Hb}$ electrophoresis was within normal

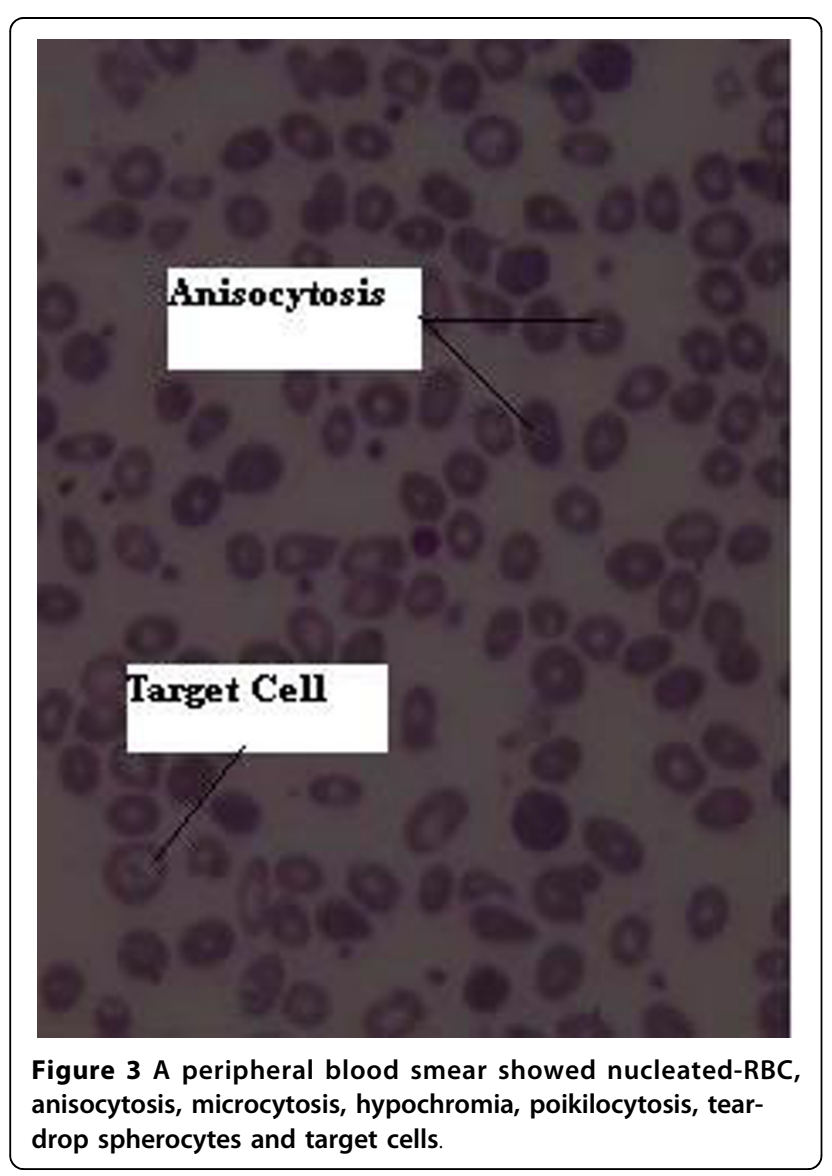

range. Her coagulation profile was intact. She had no related positive family history. She did not smoke at all. A bone marrow aspiration revealed normal appearing megakaryocytes and normoblasts with shrinked cytoplasm in the late stages of development in erythroid order and a mild shift to the left and increased lymphocyte count in white order all indicating an increase hematopoietic activity. Her hemoglobin electrophoresis was normal so a diagnosis of thalassemia was ruled out. Her serum erythropoietin level was $11 \mathrm{mU} / \mathrm{ml}$, which was normal. An arterial blood gas analysis showed normal oxygen saturation and no hypoxia.

A two-week steroid therapy was of no benefit and her neurologic status deteriorated, so we planned to perform a surgical decompression. Since she manifested dominantly with lower motor neuron problems and the dorsal lesion was very extensive with a mild compressive effect over the cord, we decided to decompress the lumbar area. She was positioned prone and a bilateral laminectomy from L2 to S1 was performed. The extensive dark-grayish epidural lesion had a fragile consistency. We removed as much of the lesion as possible. The thecal sac and the roots were successfully decompressed. Microscopic studies showed considerable replacement of epidural fat with hematopoietic cells, predominantly from erythroid and granulocytic clones and also scattered megakaryocytes (Figure 4).

The adjuvant treatment continued with whole-spine radiotherapy, which was accompanied by her gradual recovery from paraparesis. Follow-up contrast studies undertaken every six months showed no evidence of any recurrence.

Twenty-one months after her first visit, she came back complaining of headaches and visual blurring beginning

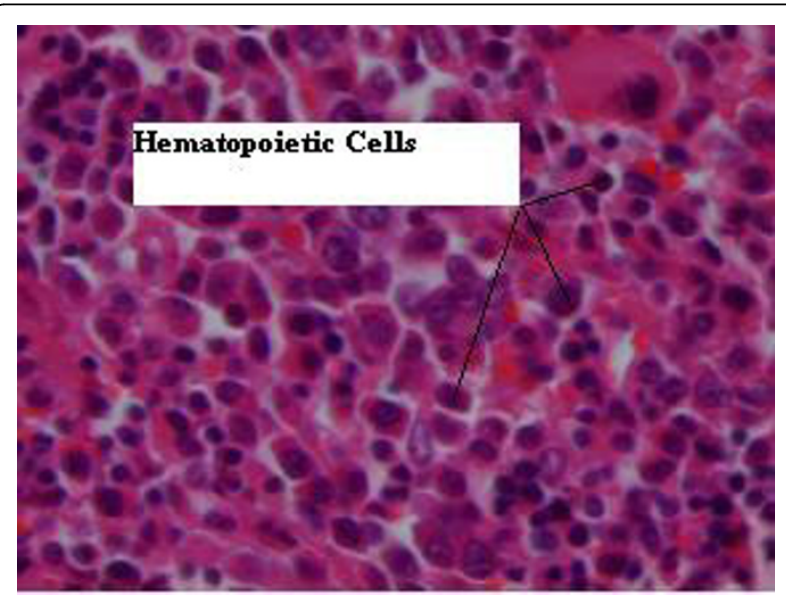

Figure $4 \mathrm{~A}$ histopathologic view of the resected epidural lesion, showing considerable replacement of epidural fat with hematopoietic cells predominantly from erythroid and granulocytic clones and also scattered megakaryocytes 
two weeks previously. A clinical assessment revealed a grade 4 papilledema and mild paraparesis (muscle strength $=4 / 5$ ) associated with generalized hyperreflexia and extensor plantar reflexes.

A whole axis contrast-enhanced magnetic study revealed a large extra-axial mass in her right frontal area, causing more than $10 \mathrm{~mm}$ midline shift, and a smaller mass in her left frontal area. The lesion became enhanced after a contrast injection and showed a dural tail sign. Patchy-enhancing foci was also evident in the meninges (Figure 5). A spine study was negative except for post-operative and post-radiation changes. A paraclinical study only showed mild thrombocytopenia and mild leukocytosis.

Her red blood cell count was $4.76 \times 10^{6} / \mathrm{mm}^{3}$; her hemoglobin level was $11.9 / \mathrm{dl}$; her hematocrit level was $37.6 \%$; her mean corpuscular volume was $79 \mathrm{fl}$; her mean corpuscular hemoglobin level was $23 \mathrm{pg}$; her mean corpuscular hemoglobin concentration was $29^{\mathrm{g}} / \mathrm{dl}$; her platelet count was $78 \times 10^{3} / \mathrm{mm}^{3}$; her white blood cell count was $32.4 \times 10^{3} / \mathrm{mm}^{3}$; the percentage of polymorphonuclear cells in her blood was $71 \%$; the percentage of lymphocytes was $13 \%$; the percentage of monocytes was $5 \%$; the percentage of myelocytes was $1 \%$; the percentage of stab cells was $9 \%$; and the percentage of metamyelocytes was $1 \%$.

She underwent a platelet transfusion because of her thrombocytopenic state. According to consultation with the hematologist, her thrombocytopenia was explained by the hypersplenism.

A course of steroid therapy was performed but it was unsuccessful. She showed signs of increased intracranial pressure, so we decided to proceed with surgery.
To reduce the intracranial pressure, a right frontoparietal craniotomy and resection of the larger lesion was performed, which was accompanied with duraplasty to improve her clinical condition. During the operation, the mass appeared dark and vascular just below the dura mater and adhering to, and in some places invading, the pia matter. Immediately after the operation, she became hemiparetic on her left side and a CT scan showed severe edema compressing her right ventricle and no evidence of hemorrhage. The medical treatment for edema was initiated but in the second post-operative day, she became hemiplegic on her left side. The hemiplegia gradually and slowly improved, although modestly. A microscopic study of the lesion indicated erythroid, myeloid and megakaryocytic proliferation indicating EMH. After consultation with hematologists and oncologists, she was sent for cranial radiation. In her follow-up visit, six months after admission, there was no evidence of any radiologic recurrence of the supratentorial lesion and the patient's condition had improved. The follow-up visits continued every six months and after two years there had been no evidence of any radiologic or clinical recurrence and the patient's condition had improved.

\section{Discussion}

Involvement of the neuroaxis due to extramedullary hematopoiesis is not common. The reported cases occurred due to some major hematologic disorders such as myelodysplastic syndromes, acute myelogenic leukemia or thalassemia major [1,2]. In very rare cases, these lesions have also been seen mixed with some neoplasms including meningioma, pilocytic astrocytoma or

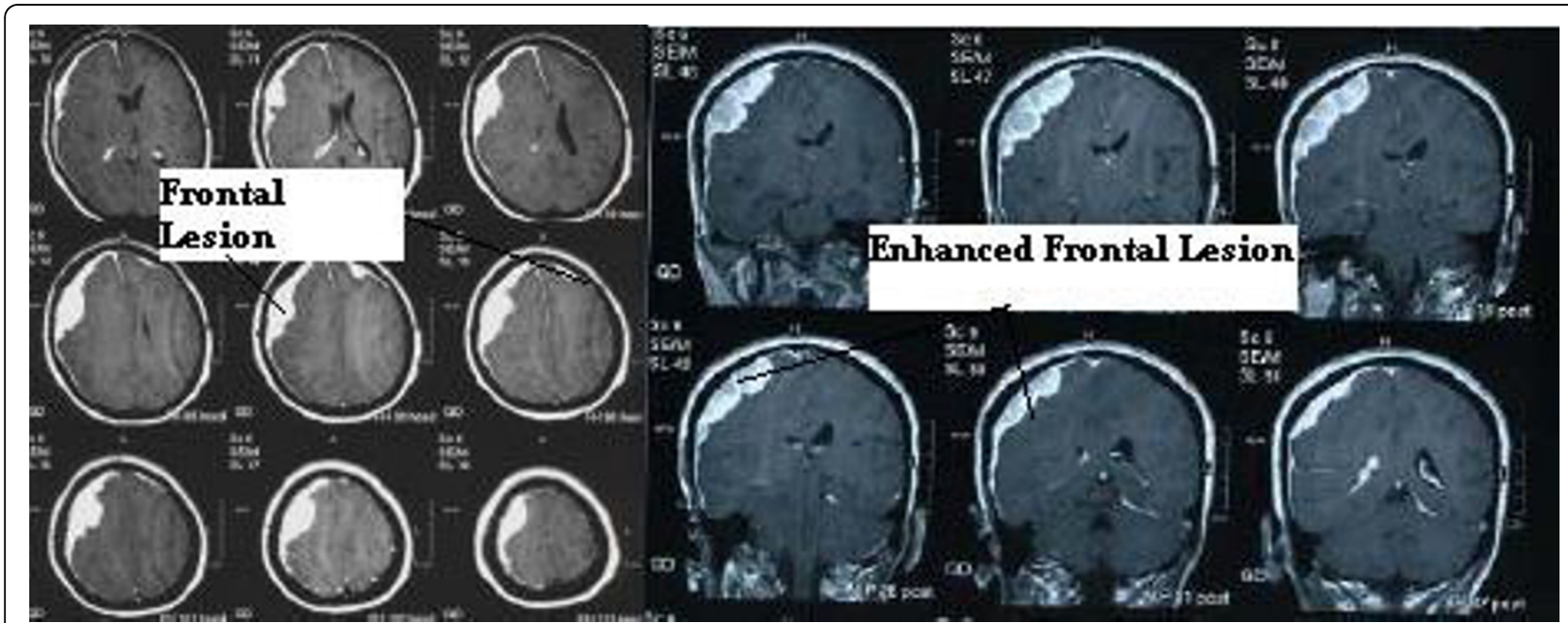

Figure $5 \mathrm{~A}$ brain MRI of the patient performed during her second admission showed an extradural lesion in the right frontoparietal region and a smaller lesion in the left frontal region (left) which became enhanced after a contrast injection (right). 
hemangioblastoma [1-3]. The low incidence of EMH in CNS indicates that cells with hematopoietic potential find little supporting environment in CNS [3]. To the best of our knowledge, we document the first reported case of sacral, lumbar, thoracic and cranial involvement in the same patient, which seems remarkable. Involvement of neuraxis in EMH is rare and most reported cases are intracranial [1-9].

Suggested treatments for these lesions include surgical removal and a combination of chemo-and radiotherapy in cases with hematologic malignancies, and blood transfusion when the underlying cause is thalassemia major, myelofibrosis or myelodysplastic syndromes.

At the first presentation of our case report, her hemoglobin level was high. She had no prior history of smoking. Her erythropoietin level and arterial blood gas analysis were normal. A bone marrow biopsy did not indicate myelofibrosis or a solid cancer metastasis and she has been disease free for two years, so these diagnoses were ruled out. We did not find the cause of her high hemoglobin level; however, it was not seen in the follow-up laboratory tests.

In our experience, she showed no sign of anemia to justify transfusion and surgical resection was considered necessary on both occasions due to the significant compressive effect and her deteriorating condition. Radiation as a treatment modality caused the thoracic lesion to disappear and there has been no evidence of any recurrence during follow-up checks. An increase of cerebral edema after surgical resection of these lesions has been reported by Gregorios et al. although EMH has occurred in a malignant meningioma in his report [8].

\section{Conclusions}

Although CNS involvement in EMH is very rare, this entity deserves attention as a differential diagnosis in patients with an underlying hematologic disorder.

\section{Consent}

Written informed consent was obtained from the patient for publication of this case report and any accompanying images. A copy of the written consent is available for review by the Editor-in-Chief of this journal.

\section{Abbreviations \\ CT scan: computed tomography scan; EMH: extramedullary hematopoiesis; $\mathrm{HCT}$ : hematocrit; Hgb: hemoglobin; $\mathrm{MCH}$ : mean corpuscular hemoglobin; MCHC: mean corpuscular hemoglobin concentration; MRI: magnetic resonance imaging; Plt: platelet; RBC: red blood cell; U: unit; WBC: white blood cell.}

\section{Author details}

'Shohada Tajrish Hospital, Beheshti University of Medical Sciences, Tehran, Iran. ${ }^{2}$ Rajaie Hospital, Qazvin University of Medical Sciences, Qazvin, Iran. ${ }^{3}$ Neurofunctional Research Center, Shohada Tajrish Hospital, Beheshti University of Medical Sciences, Tehran, Iran.

\section{Authors' contributions}

ASS analyzed and interpreted the patient data regarding the lateral sacral meningocele and performed the surgery. AS performed the review of literature and was a major contributor in writing the manuscript. All authors read and approved the final manuscript.

\section{Competing interests}

The authors declare that they have no competing interests.

Received: 7 January 2009 Accepted: 12 October 2010

Published: 12 October 2010

\section{References}

1. Obara H, Nishimura S, Hayashi N, Numagami $Y$, Inue T, Kubo K, Kaimori M, Nishijima M: Intracranial granulocytic sarcoma in a patient with acute myeloid leukemia. No To Shinkei 2006, 58:797-801.

2. Beckner ME, Lee JY, Schochet SS Jr, Chu CT: Intracranial extramedullary hematopoiesis associated with pilocytic astrocytoma: a case report. Acta Neuropathol 2003, 106:584-587.

3. Roy EP, Rogers JS, Riggs JE: Intracranial granulocytic sarcoma in postpolycythemia myeloid metaplasia. South Med J 1989, 82:1564-1567.

4. Fueredi GA, Czarnecki DJ, Cooley PA: Evaluating EMP with computed tomography. Wis Med J 1989, 88:15-16.

5. Landolfi R, Colosimo C Jr, De Candia E, Castellana MA, De Cristofaro R, Trodella L, Leone G: Meningeal hematopoiesis causing exophthalmus and hemiparesis in myelofibrosis: effect of radiotherapy. A case report. Cancer 1988, 62:2346-2349.

6. Fucharoen S, Suthipongchai S, Poungvarin N, Ladpli S, Sonakul D, Wasi P. Intracranial extramedullary hematopoiesis inducing epilepsy in a patient with beta-thalassemia-hemoglobin E. Arch Intern Med 1985, 145:739-742.

7. Robitaille GA, Eisenberg M, Lehman R: Intracranial extramedullary hematopoiesis in polycythemia vera. Conn Med 1985, 49:149-151.

8. Gregorios JB, Bay JW, Dudley AW Jr: Extramedullary hematopoiesis in a malignant meningioma. Neurosurgery 1983, 13:447-451.

9. Kandel RA, Pritzker KP, Gordon AS, Bilbao JM: Extramedullary hematopoiesis simulating parasagittal meningioma. Can J Neurol Sci 1982, 9:49-51.

doi:10.1186/1752-1947-4-319

Cite this article as: Seddighi and Seddighi: Extramedullary hematopoiesis presenting as a compressive cord and cerebral lesion in a patient without a significant hematologic disorder: a case report. Journal of Medical Case Reports 2010 4:319.

\section{Submit your next manuscript to BioMed Central} and take full advantage of:

- Convenient online submission

- Thorough peer review

- No space constraints or color figure charges

- Immediate publication on acceptance

- Inclusion in PubMed, CAS, Scopus and Google Scholar

- Research which is freely available for redistribution 